\title{
DESIGN OF THE EXTRACTION SYSTEM OF THE SUPERCONDUCTING ECR ION SOURCE VENUS*
}

\author{
M.A. Leitner ${ }^{* *}$, D.C. Wutte, C.M. Lyneis \\ LBNL, Berkeley, CA 94720, USA
}

\begin{abstract}
A new, very high magnetic field superconducting ECR ion source, VENUS, is under construction at the LBNL 88-Inch Cyclotron [1,2]. The paper describes the VENUS extraction system and discusses the ion beam formation in the strong axial magnetic field $(3 \mathrm{~T})$ of the ECR ion source. Emittance values as expected from theory, which assumes a uniform plasma density across the plasma outlet hole, are compared with actual measurements from the AECR-U ion source. Results indicate that highly charged heavier ions are concentrated on the source axis. They are extracted from an "effective" plasma outlet hole, whose smaller radius must be included in ion optics simulations.
\end{abstract}

\section{SOURCE DESIGN}

The magnet structure of VENUS, which consists of three superconducting axial coils and six superconducting radial coils in a sextupole configuration, will generate a maximum axial field of 4 Tesla at the injection side and 3 Tesla at the extraction side. The maximum sextupole field is 2 Tesla at the plasma chamber wall. These high magnetic fields are designed to allow operation with $28 \mathrm{GHz}$ ECR-heating frequency, twice the frequency of current ECR sources. Since the plasma density is expected to scale as frequency squared we anticipate to extract significantly more intense (factor of 4 to 5) ion beams than presently achievable by ECR ion sources. To transport these intense heavy ion beams the extraction system and mass-analyzing system of the ion source is designed for a proton-equivalent current of $25 \mathrm{~mA}$ at $30 \mathrm{kV}$ extraction voltage.

\section{EXTRACTION}

Figure 1 shows the magnetic field distribution of the ion source, its extraction region, and a subsequent solenoid lens for matching the ion beam to the analyzing magnet. Extraction and beam formation take place in a strong (up to 3T) axial magnetic field, which leads to significantly different focusing properties for the different ion masses and charge states of the extracted beam. Due to the large size of the cryostat (including the iron yoke) the extracted ion beam has to be transported without additional focusing element for $\sim 48 \mathrm{~cm}$. In this region the magnetic field drops from 3 to zero Tesla inducing a strong

\footnotetext{
* This work was supported by the Director, Office of Energy Research, Office of High Energy and Nuclear Physics, Nuclear Physics Division of the U.S. Department of Energy under Contract DE AC03-76SF00098.

** MLeitner@lbl.gov; http://ecrgroup.lbl.gov
}

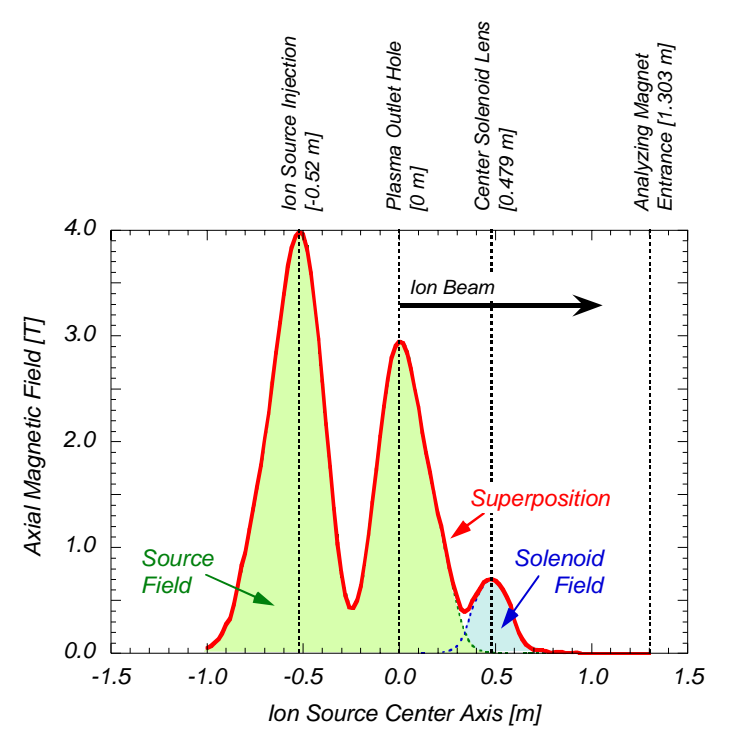

Figure 1: Magnetic field distribution of the VENUS ion source, its extraction region, and a subsequent solenoid lens for matching the ion beam to the analyzing magnet.

azimuthal momentum in the ion beam, which leads to a considerable emittance increase.

\subsection{Influence of the magnetic field}

The VENUS extraction region has been simulated and optimized with IGUN [3], an axi-symmetric ion optics code for plasma extraction allowing the inclusion of multiple charge states with different space charge contributions. A charge state distribution (from the existing AECR-U ion source) scaled up to the expected higher beam intensities of $25 \mathrm{~mA}$ proton-equivalent current has been used as input to the simulations.

Calculations have been performed by simulating only a single charge state of interest. The space charge contribution of the whole charge state distribution has been normalized to the equivalent current of the simulated charge state. This method is explained in reference [4]. It has been shown [4] that such a procedure allows an accurate extraction simulation in the presence of a strong axial magnetic field. In addition, simulations using multiple charge states have been performed to investigate the space charge effects due to the mass dependent focusing action of the magnetic field.

For an ECR extraction system two main contributions to the ion beam emittance have to be considered: (1) the ion beam temperature and (2) the induced beam rotation due to the decreasing axial magnetic field. 
The emittance due to the ion temperature can be given by assuming a Maxwellian temperature distribution inside the plasma (see [5]):

$$
\varepsilon_{T E M P}^{x x^{\prime}-r m s-n o r m}=0.016 \cdot r \cdot \sqrt{\frac{k T_{i}}{M / Q}}
$$

$\{\varepsilon \ldots$ normalized $\mathrm{x}$-x' $\mathrm{rms}$ emittance $[\pi \mathrm{mm} \mathrm{mrad}]$; $r$...plasma outlet hole radius $[\mathrm{mm}] ; k T_{i} \ldots$ ion temperature $[\mathrm{eV}] ; M / Q \ldots$ amu/charge [dim.less] $\}$

Under the assumption of a uniform plasma density across the plasma outlet hole the emittance due to the beam rotation induced by the decreasing ion source magnetic field is described by the Busch's theorem (see [6], assuming $\varepsilon^{100 \%}=5 \cdot \varepsilon^{r m s}$, a waterbag distribution)

$$
\varepsilon_{M A G}^{x x^{\prime}-r m s-n o r m}=0.032 \cdot r^{2} \cdot B_{0} \cdot \frac{1}{M / Q}
$$

$\{\varepsilon \ldots$...normalized $\mathrm{x}$-x' $\mathrm{rms}$ emittance $[\pi \mathrm{mm} \mathrm{mrad}]$; $r$...plasma outlet hole radius $[\mathrm{mm}] ; B_{0} \ldots$ axial magnetic field [T]; $M / Q \ldots$ amu/charge [dim.less] $\}$

The beam rotation due to the decreasing magnetic ECR ion source field becomes the dominating contribution to the ion beam emittance when following condition, as derived by combining equation (1) and (2), is satisfied:

$$
B_{0} \cdot r \geq 0.5 \cdot \sqrt{k T_{i}} \cdot \sqrt{M / Q}
$$

$\left\{B_{0} \ldots[\mathrm{T}] ; r_{\ldots} \ldots[\mathrm{mm}] ; k T_{i} \ldots[\mathrm{eV}] ; M / Q \ldots[\right.$ dim.less $\left.]\right\}$

Table 1 summarizes the minimum magnetic field values where the emittance starts to be entirely dominated by the ion beam rotation. A plasma outlet hole radius of $4 \mathrm{~mm}$ and an ion temperature of $0.3 \mathrm{eV}$ has been assumed. It is evident that the magnetic field is the main contribution to the ion beam emittance in most ECR ion sources.

Figure 2 demonstrates this behavior for a ${ }^{238} \mathrm{U}^{20+}$ ion beam simulated through the VENUS extraction system until the axial magnetic field drops to zero $(\mathrm{z}=0.6 \mathrm{~m}$ in figure 1). The total extracted current is $25 \mathrm{~mA}$ (proton equivalent), the ${ }^{238} \mathrm{U}^{20+}$ current is $211 \mathrm{e} \mu \mathrm{A}(10.6 \mathrm{p} \mu \mathrm{A})$. The extraction voltage is $30 \mathrm{kV}$, the plasma outlet hole

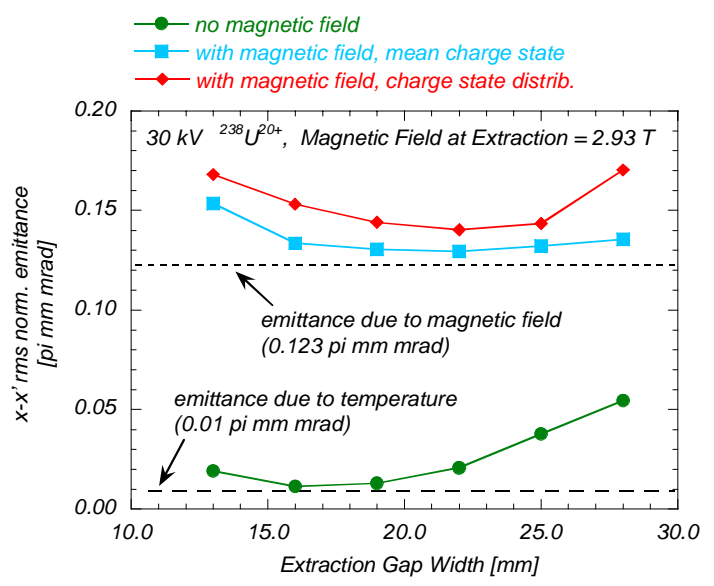

Figure 4: Ion beam emittance vs. extraction gap. Results of an IGUN simulation without and with axial magnetic field present.
Table 1: Magnetic field values where the emittance starts to be entirely dominated by the ion beam rotation

\begin{tabular}{|l|l|}
\hline $\mathrm{M} / \mathrm{Q}=1$ & $\mathrm{~B}_{0}>0.07 \mathrm{~T}$ \\
\hline $\mathrm{M} / \mathrm{Q}=5$ & $\mathrm{~B}_{0}>0.15 \mathrm{~T}$ \\
\hline $\mathrm{M} / \mathrm{Q}=10$ & $\mathrm{~B}_{0}>0.22 \mathrm{~T}$ \\
\hline $\mathrm{M} / \mathrm{Q}=30$ & $\mathrm{~B}_{0}>0.38 \mathrm{~T}$ \\
\hline $\mathrm{M} / \mathrm{Q}=100$ & $\mathrm{~B}_{0}>0.69 \mathrm{~T}$ \\
\hline
\end{tabular}

radius is $4 \mathrm{~mm}$. If the extraction gap electric field strength is matched to the plasma meniscus the norm. rms $\mathrm{x}-\mathrm{x}$ ' emittance approaches the minimum theoretical value of $0.01 \pi \mathrm{mm}$ mrad as determined by the ion temperature (see equation 1) if no magnetic field is present in the simulation.

With an axial magnetic field present, which peaks at the plasma outlet hole at 2.93 Tesla and drops to zero Tesla according to figure 1 , the norm. rms $\mathrm{x}-\mathrm{x}$ ' emittance increases significantly to $\sim 0.13 \pi \mathrm{mm}$ mrad. This value is consistent with equation (2). The simulations with the magnetic field present were performed with only a single mean charge state (with normalized currents according to [4]) as well as with multiple charge states. Both results are comparable in the range of uncertainty associated with such simulations as well as with actual emittance measurements.

The emittance due to the magnetic field at the extraction is dependent on the $\mathrm{M} / \mathrm{Q}$ ratio as indicated in equation (2). Figure 3 plots the emittance versus M/Q for VENUS extraction conditions (plasma outlet hole radius: $4 \mathrm{~mm}$; axial magnetic field at extraction: $2.93 \mathrm{~T}$; extraction voltage: $30 \mathrm{kV}$; proton-equiv. total current: $25 \mathrm{~mA}$ ). The data points in the figure have been derived from IGUN simulations. The simulated emittances agree with the theoretical emittance values obtained from equation (2). The emittance contribution due to the thermal energy of the ions is also indicated in the figure. It is evident that the thermal energy is not important for the ion beam emittance.

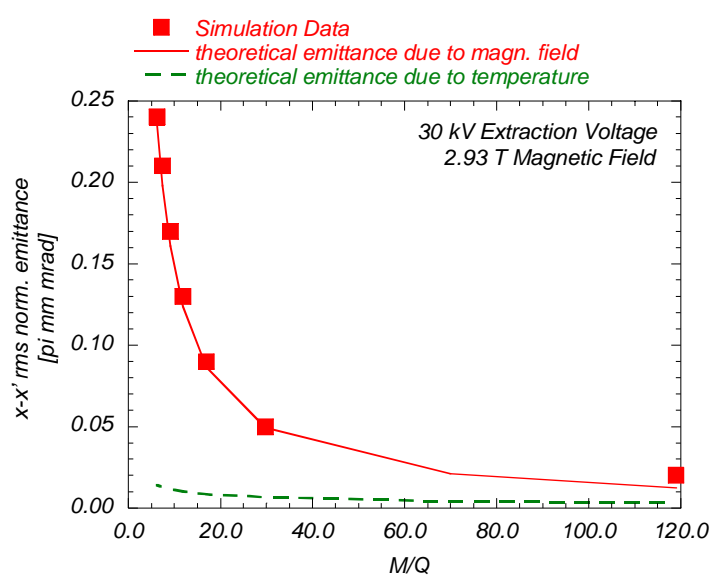

Figure 3: Simulated and calculated emittance values versus M/Q for VENUS extraction conditions. 


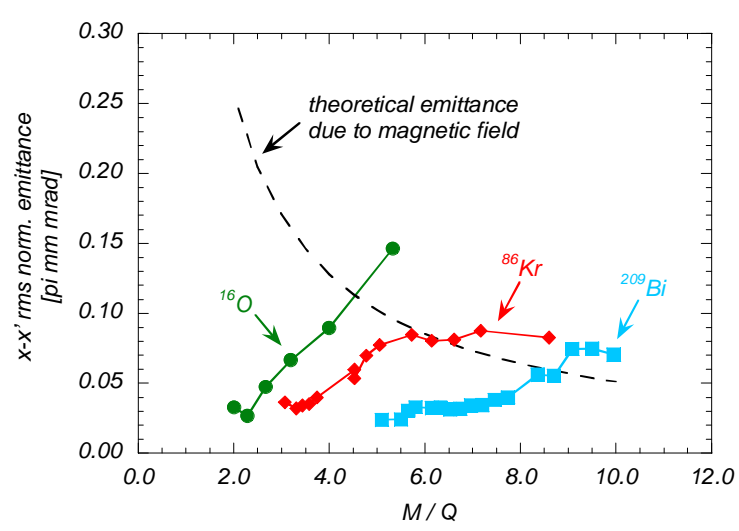

Figure 4: Emittance values for different ion beam masses and charge states as measured after the analyzing magnet of the AECR-U injector ion source at the 88-Inch Cyclotron.

\subsection{Comparison with experimental results}

Figure 4 shows emittance values for different ion beam masses and charge states as measured [7] after the analyzing magnet of the AECR-U injector ion source at the 88-Inch Cyclotron. The total extracted current is approx. equal for all the different masses $(1.5 \mathrm{~mA} \approx 1 / 4$ of the expected VENUS currents), the axial peak magnetic field at extraction was $\sim 0.9$ Tesla, the plasma outlet hole radius is $4 \mathrm{~mm}$.

The emittance values show a M/Q dependence, which is contrary to the behavior expected from equation (2). The emittance values as expected from equation (2) are indicated in the figure with a dotted line. Within a charge state distribution for a particular element the measured emittance decreases for higher charge states. In addition, the measurements indicate a strong mass dependence of the normalized emittance. For example the normalized rms emittance for $\mathrm{O}^{3+}(0.146 \pi \mathrm{mm} \mathrm{mrad})$ is about 6 times higher than for $\mathrm{Bi}^{38+}(0.024 \pi \mathrm{mm} \mathrm{mrad})$. This behavior cannot be explained with ion optical considerations but must be due to plasma physics and ion production processes, e.g. radial transport phenomena for different ions and electrons. The highly charged heavier ions

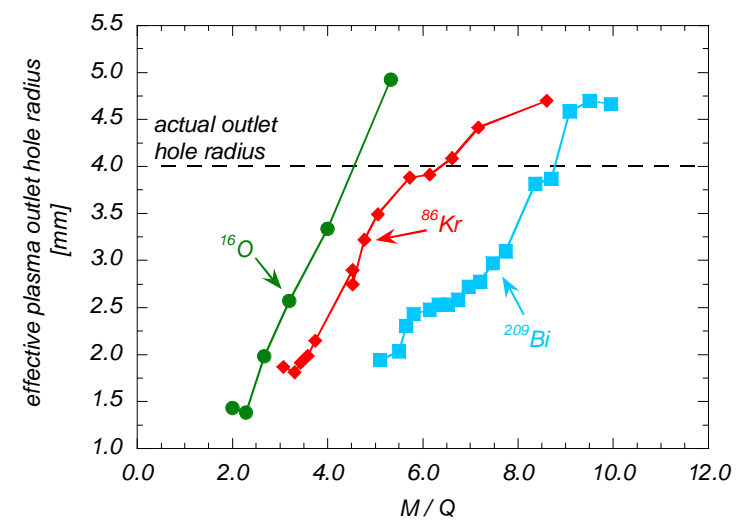

Figure 5: Effective extraction radii as defined by equation (4) for different ions. appear to be concentrated more on the axis of the ion source, whereas the low charge state ions could also be produced in the outer shell of the ECR plasma.

This is in agreement with an early observation [8] that the extracted currents for high charge state ions decrease less than for the low charge state ions after reduction of the plasma outlet hole diameter. Therefore, an effective outlet hole radius may be defined for the higher charge states and heavier masses by comparing the measured emittance values with the theoretical emittance value computed from equation (2):

$$
r_{\text {eff }}=\sqrt{r_{a c t}^{2} \cdot \frac{\varepsilon_{a c t}}{\varepsilon_{t h}}}
$$

$\left\{r_{\text {eff }} \ldots\right.$ effective radius; $r_{a c t} \ldots$ actual plasma outlet hole radius; $\varepsilon_{a c t} \ldots$ measured emittance value; $\varepsilon_{t h} \ldots$ theoretical emittance value according to equation (2) \}

Such effective radii for different ions are plotted in figure 5 for the AECR-U ion beams taken from figure 4 . The concentration of the high charge state heavy ions on the source axis is clearly visible. The larger extraction radii compared to the actual outlet hole radius for the low charge state ions are most probably caused by beam transport imperfections, e.g. aberrations or unmatched extraction conditions.

\section{CONCLUSION}

Because of the induced beam rotation due to the decreasing axial magnetic field of the ion source the emittance values for high charge state heavy ion beams extracted out of an ECR ion source would be huge. Experimental results indicate that plasma transport and ionization processes lead to a concentration of higher charge states and heavier ions on the ion source axis. Therefore, these ions are extracted from a smaller "effective" plasma outlet hole, whose radius must be used in the ion optics simulations. The resultant emittance values are then well below requirements imposed by current accelerators and future accelerator projects.

\section{REFERENCES}

[1] C.M. Lyneis, M.A. Leitner, D. Wutte, C.E. Taylor and S.R. Abbott, Proc. 2001 International Conference on Cyclotrons and their Applications, May 12-17 2001, East Lansing, Michigan

[2] M.A. Leitner, C.M. Lyneis, D. Wutte, C.E. Taylor and S.R. Abbott: "Construction of the Superconducting ECR Ion Source VENUS", to be published in Physica Scripta, 2001

[3] R. Becker, Proc. Sixth European Particle Accelerator Conference, June 22 - 26 1998, Stockholm, Sweden

[4] D. Wutte, M.A. Leitner, C.M. Lyneis, C.E. Taylor and Z.Q. Xie, Proc. Eighth International Conference on Heavy-Ion Accelerator Technology, October 5-9 1998, Argonne, Illinois

[5] I.G. Brown: "The physics and technology of ion sources", 1998: Wiley, New York, pages 93f

[6] A. Septier: "Focusing of charged particles", Vol. II, 1967 Academic Press, New York, pages 76ff

[7] D. Wutte, M.A. Leitner and C.M. Lyneis: "Emittance measurements for high charge state ion beams extracted from the AECR-U ion source", to be published in Physica Scripta, 2001

[8] D.J. Clark: Proc. International Conference on ECR Ion Sources, 1987, East Lansing, Michigan 